\title{
On Consensus of Star-Composed Networks with an Application of Laplacian Spectrum
}

\author{
Da Huang, ${ }^{1,2}$ Haijun Jiang, ${ }^{1}$ Zhiyong Yu, ${ }^{1}$ Qiongxiang Huang, ${ }^{1}$ and Xing Chen ${ }^{2}$ \\ ${ }^{1}$ College of Mathematics and System Sciences, Xinjiang University, Urumqi, Xinjiang 830046, China \\ ${ }^{2}$ Xinjiang Institute of Engineering, Urumqi, Xinjiang 830023, China \\ Correspondence should be addressed to Haijun Jiang; jianghaijunxju@163.com
}

Received 6 September 2016; Revised 8 November 2016; Accepted 26 December 2016; Published 5 March 2017

Academic Editor: Seenith Sivasundaram

Copyright (c) 2017 Da Huang et al. This is an open access article distributed under the Creative Commons Attribution License, which permits unrestricted use, distribution, and reproduction in any medium, provided the original work is properly cited.

\begin{abstract}
In this paper, we mainly study the performance of star-composed networks which can achieve consensus. Specifically, we investigate the convergence speed and robustness of the consensus of the networks, which can be measured by the smallest nonzero eigenvalue $\lambda_{2}$ of the Laplacian matrix and the $H_{2}$ norm of the graph, respectively. In particular, we introduce the notion of the corona of two graphs to construct star-composed networks and apply the Laplacian spectrum to discuss the convergence speed and robustness for the communication network. Finally, the performances of the star-composed networks have been compared, and we find that the network in which the centers construct a balanced complete bipartite graph has the most advantages of performance. Our research would provide a new insight into the combination between the field of consensus study and the theory of graph spectra.
\end{abstract}

\section{Introduction}

The research on consensus problems of networks has received much attention in the last decade. There are many potential applications in several aspects related to consensus such as sensor networks, formation control, and decision-making. It is well known that the first milestone of development for network study is related to the famous Euler graph, and then the application of graph theory came into many fields of network study in the past decades. One advantage of studying network through abstract graph is that it can let us compare the differences of properties of topologies between different networks in a more convenient way.

For the purpose of achieving consensus, much of the relationships among agents are encoded by the communication graph of the system, and it is well known that the coordination problem can be connected with the Laplacian matrix of the communication graph ([1-3]). This fact can let us take graph theory as a more efficiency tool to study the consensus problem. Recently, there are many articles on consensus or other network properties related to the eigenvalue of Laplacian matrix of graph $([1,2,4-8])$.
The network coherence of fractal network is studied in [6] by using properties of Laplacian eigenvalues. Reference [7] has studied consensus problems in a family of tree networks and investigated first- and second-order consensus characterized by Laplacian spectrum. In [5], the authors have studied the Laplacian spectra of recursive treelike smallworld polymer networks and analysed the solution with some related application. In [2], the authors have investigated how the robustness depends on the properties of the Laplacian eigenvalues of graphs and give a derivation for the convergence speed and the $\mathrm{H}_{2}$ norms of some specific graphs.

In [1], Olfati-Saber and Murray have showed that the Fiedler eigenvalue $\lambda_{2}$ of an undirected (or directed) graph can characterize the convergence speed of consensus problem. The article gives an idea that a network with dense interconnections solves an agreement problem faster than a connected but sparse network is briefly considered and can be further discussed. Since most Laplacian eigenvalues of graphs can not be determined precisely, we can not analyse the convergence speed or other network performances of most graphs in a quantitative way. It is well known that the Laplacian spectrum of star graph has already been determined, and star networks 
are one of the most common computer network topologies. Star-related structure of network are widely considered in many fields like the consensus problem and synchronization problem, and so on $([2,9-15])$.

Inspired by this notion, we consider some meaningful specific networks of certain topologies that are related to star graphs. The star network can be viewed as point-to-multipoint communication system, and the star-composed network can be comprehended as adding communication links among centers on the basis of star networks. It makes sense that all the star topologies have connection with each other only through their center nodes because of the role of their position, and it is natural that the leaf nodes do not have the authorities to communicate among themselves.

The consensus in this article means that each agent reaches an agreement by local communication. Our findings bring a better perspective into the combination between consensus problems and the field of graph theory, and our work makes further efforts to use the theory of graph spectra for studying performance of consensus, where the "performance" in this article mainly refers to the convergence speed and robustness of consensus. Due to the chosen undirected graphs, we study the convergence speed and robustness of consensus to communication noise with an application of Laplacian spectrum of corona $([16,17])$ of two graphs, and find that the method of constructing networks by using the notion of corona of two graphs can generate a class of polymer small-world network ([5]).

The paper is organized as follows. In Section 2, some notations on graph theory are summarized and the relations between performance and Laplacian eigenvalues are explained. In Section 3, the constructions of star-composed networks and main results on Laplacian eigenvalues are given. In Section 4, combined with theorem of algebraic graph theory, we analyse the simulation results in Section 3 and make the comparison of performances for the three classes of networks.

\section{Preliminaries}

2.1. Graph Theory and Notations. Throughout this paper, we consider only simple undirected graphs even if without special explanation, where simple graph means the graph has no self-loops or parallel edges ([18]). It is well known to us that the networked system comprises mainly two factors, which are the dynamics of agents and the interaction topology among the nodes. Thus, when the dynamics are given, the network can be simplified as a graph. For convenience of expression, the terms, topology, and graph, vertex, and node will be used without distinction in this paper. We denote a complete graph of $n$ vertices by $K_{n}$ and denote a star graph with $k$ leaves by $S_{k}$, where the leaf refers to the vertex of degree one. $P_{k}$ is defined to be the empty graph with $k$ vertices, where empty graph refers to the graph without edges among all the nodes of the graph. Let $G$ be a graph with vertex set $V=\left\{v_{1}, v_{2}, \ldots, v_{N}\right\}$, and the edge set of $G$ is defined to be $\mathscr{E}=\{(i, j) \mid i, j=1,2, \ldots, N ; i \neq j\}$. The adjacency matrix of $G$, denoted by $A(G)$, is defined as $A(G)=\left[a_{i j}\right]_{N}$, where $a_{i j}$ is the weight of the edge $(i, j)$. An undirected graph is a graph in which edges have no orientation. To an undirected graph, we can see that $(i, j)$ and $(j, i)$ are the same edge in $\mathscr{E}$, and $a_{i j}=$ $a_{j i}$. An edge $(i, j)$ in a weighted undirected graph denotes that the information transmission between two connected nodes is bidirectional. Since we consider mainly the influence of network structure, all the edges in our networks are supposed to have 0 - 1 weights; that is, $a_{i j}=\left\{\begin{array}{l}1,(i, j) \in \mathscr{E} ; \\ 0,(i, j) \notin \mathscr{E} \text {. }\end{array}\right.$. The Laplacian matrix of $G$, denoted by $L(G)$, is defined as $D(G)-A(G)$ and can be simplified by $L=D-A$, where $D(G)$ is the diagonal degree matrix of $G$ defined by $D(G)=\operatorname{diag}\left(d_{1}, d_{2}, \ldots, d_{N}\right)$ with $d_{i}=\sum_{j \neq i} a_{i j}$. The degree of a node in an undirected graph $G$ is the number of edges that link to the node. The minimum degree of $G$ is the minimum number of degrees of all the nodes in $G$ and is denoted by $\delta(G)$. Note that if a graph is undirected, then its adjacency matrix and Laplacian matrix are both symmetric. The Laplacian spectrum of $G$ is defined as $\operatorname{spec}(L(G))=\left(\begin{array}{cccc}\lambda_{1}(G) & \lambda_{2}(G) & \cdots & \lambda_{p}(G) \\ l_{1} & l_{2} & \ldots & l_{p}\end{array}\right)$, where $\lambda_{1}(G)<$ $\lambda_{2}(G)<\cdots<\lambda_{p}(G)$ are the eigenvalues of $L(G)$ and $l_{1}, l_{2}$, $\ldots, l_{p}$ are the multiplicities of these eigenvalues. The spectrum can be also simply denoted by a sequence of eigenvalues $($ see $[16,17,19]): S(L(G))=\left(\lambda_{1}, \lambda_{2}, \ldots, \lambda_{N}\right)$, where $\lambda_{1} \leq \lambda_{2} \leq$ $\cdots \leq \lambda_{N}$ are the Laplacian eigenvalues of $G$. For any undirected graph, it is well known that $\lambda_{1}=0$ is an eigenvalue of $L(G)$ associated with the eigenvector $\mathbf{1}=(1,1, \ldots, 1)^{T}$ and $L(G)$ is a positive semidefinite matrix.

Definition 1 (see [1] (mirror graph)). Let $G=(V, \mathscr{E}, A)$ be a weighted digraph. Let $\overline{\mathscr{E}}$ be the set of reverse edges of $G$ obtained by reversing the order of nodes of all the pairs in $\mathscr{E}$. The mirror of $G$ denoted by $\widehat{G}$ is an undirected graph in the form $\widehat{G}=(V, \widehat{\mathscr{E}}, \widehat{A})$ with the same set of nodes as $G$, the set of edges $\widehat{\mathscr{E}}=\mathscr{E} \cup \overline{\mathscr{E}}$, and the symmetric adjacency matrix $\widehat{A}=\left[\widehat{a}_{i j}\right]$ with elements

$$
\widehat{a}_{i j}=\widehat{a}_{j i}=\frac{a_{i j}+a_{j i}}{2} \geq 0 .
$$

Definition 2 (see [1] (balanced graph)). The node $v_{i}$ of a digraph $G=(V, \mathscr{E}, A)$ is defined to be balanced if and only if its indegree and outdegree are equal. A graph $G$ is called balanced if and only if all of its nodes are balanced, or

$$
\sum_{j} a_{i j}=\sum_{j} a_{j i} \quad \forall i .
$$

Remark 3. In graph theory, we know that an undirected connected graph $G$ can be viewed as a directed graph through the operation of replacing each edge by two opposite arcs with same weight; therefore it is strongly connected and balanced. By Theorem 4 of [1], we know that any undirected connected graph can achieve consensus. In this article, all the graphs of networks we considered is undirected and 0-1 weighted; through (1), we have $\widehat{a}_{i j}=\widehat{a}_{j i}=1$ or $\widehat{a}_{i j}=\widehat{a}_{j i}=0$; one can see that the mirror of an undirected graph is itself. Let $L_{s}=$ $\left(L+L^{T}\right) / 2$; since $\widehat{d}_{i}=\Sigma\left(\left(a_{i j}+a_{j i}\right) / 2\right)=d_{i}$, thus $\widehat{D}=D$; therefore, $L_{s}=(1 / 2)\left(L+L^{T}\right)=D-\left(A+A^{T}\right) / 2=\widehat{D}-\widehat{A}=\widehat{L}=L(\widehat{G})$. 


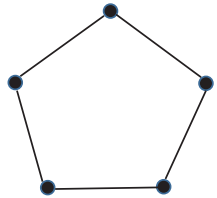

(a) $G_{1}$

(b) $G_{2}$

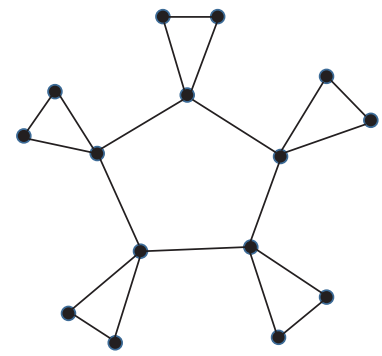

(c) $G_{1} \circ G_{2}$

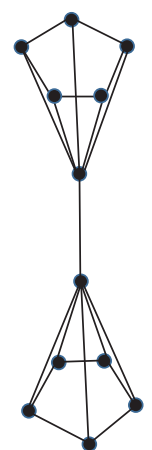

(d) $G_{2} \circ G_{1}$

FIGURE 1

Definition 4 (see [16] (the corona of two graphs)). Let $G_{1}$ and $G_{2}$ be two graphs on disjoint sets of $n$ and $k$ vertices, respectively. The corona $G_{1} \circ G_{2}$ of $G_{1}$ and $G_{2}$ is defined as the graph obtained by taking one copy of $G_{1}$ and $n$ copies of $G_{2}$ and then joining the $i$ th vertex of $G_{1}$ to every vertex in the $i$ th copy of $G_{2}$.

Example 5. Let $G_{1}=C_{5}$, the cycle of 5 nodes, and $G_{2}=K_{2}$. The two different coronas $G_{1} \circ G_{2}$ and $G_{2} \circ G_{1}$ are shown in Figures $1(\mathrm{c})$ and $1(\mathrm{~d})$.

Lemma 6 (see $[16,17]$ ). Let $G_{1}$ be any graph with $n_{1}$ vertices and $m_{1}$ edges and $G_{2}$ be any graph with $n_{2}$ vertices and $m_{2}$ edges. Suppose that $S\left(L\left(G_{1}\right)\right)=\left(\mu_{1}, \mu_{2}, \ldots, \mu_{n_{1}}\right)$ and $S\left(L\left(G_{2}\right)\right)=\left(\delta_{1}, \delta_{2}, \ldots, \delta_{n_{2}}\right)$. Then the Laplacian spectrum of $G_{1} \circ G_{2}$ is given by

(i) two multiplicity one eigenvalues $\left(\left(\mu_{i}+n_{2}+1\right) \pm\right.$ $\left.\sqrt{\left(\mu_{i}+n_{2}+1\right)^{2}-4 \mu_{i}}\right) / 2 \in S\left(L\left(G_{1} \circ G_{2}\right)\right)$ for each eigenvalue $\mu_{i}\left(i=1,2, \ldots, n_{1}\right)$ of $S\left(L\left(G_{1}\right)\right)$;

(ii) $\delta_{j}+1 \in \operatorname{spec}\left(L\left(G_{1} \circ G_{2}\right)\right)$ with multiplicity $n_{1}$ for every eigenvalue $\delta_{j}\left(j=2, \ldots, n_{2}\right)$ of $S\left(L\left(G_{2}\right)\right)$.

Definition 7 (see [1] (communication cost)). For 0-1 weighted undirected graphs, the communication cost is defined by

$$
C=\sum_{i, j=1}^{N} a_{i j}=|\mathscr{E}| .
$$

Definition 8 (network density). The density $\rho$ of a network which contains $N$ nodes is defined as the quotient of the number of edges $M$ that exist in the network and the maximum possible number of edges in the network. For undirected network, we have

$$
\rho=\frac{M}{(1 / 2) N(N-1)} .
$$

2.2. Relations between the Performance and Laplacian Spectrum. When we discuss the convergence speed of consensus, the dynamic of each node in the network is defined as follows:

$$
\dot{x}=-L x,
$$

where $\dot{x}_{i}=\sum_{v_{j} \in N_{i}} a_{i j}\left(x_{j}-x_{i}\right), N_{i}$ is the set of neighbours of $v_{i}, x_{i}$ denotes the value of node of $v_{i}, x=\left(x_{1}, x_{2}, \ldots, x_{N}\right)^{T}$ is the state vector of network, and $L$ is the Laplacian matrix of graph. The value of node represents physical quantities like position, attitude, and so on. We can comprehend the graph of the network as an information flow. For an undirected connected graph $G$, the following well known property holds ([20]):

$$
\min _{x \neq 0,1^{T} x=0} \frac{x^{T} L x}{\|x\|^{2}}=\lambda_{2}(L) .
$$

The relation between convergence speed of consensus and $\lambda_{2}$ of Laplacian matrix of graph can be understood by the following derivation on protocol (5).

By Theorem 6 of [1], we know that, for networks with balanced information flow, since $\mathbf{1}^{T} x^{\prime}=-\mathbf{1}^{T} L x=0$ and $\sum_{i=1}^{N} x_{i}=C$ is a constant, we have that $\alpha=\operatorname{Ave}(x)=C / N$ is an invariant quantity, and $x$ can be decomposed by the following equation:

$$
x=\alpha \mathbf{1}+\delta,
$$

where $\delta \in R^{N}$ and $\delta$ is the disagreement vector which evolves according to the dynamic

$$
\dot{\delta}=-L \delta
$$

It follows from (7) that $\sum_{i=1}^{N} x_{i}=N \alpha+\sum_{i=1}^{N} \delta_{i}$; therefore $\sum_{i=1}^{N} \delta_{i}=0$.

Let $V(\delta)=(1 / 2)\|\delta\|^{2}$, by (6) and Remark 3, we have $\dot{V}=$ $-\delta^{T} L \delta=-\delta^{T} L_{s} \delta=-\delta^{T} \widehat{L} \delta \leq-\lambda_{2}(L)\|\delta\|^{2}=-2 \kappa V(\delta)<0$, $\forall \delta \neq 0$. And because $\int_{0}^{t}\left(V^{\prime}(\delta) / V(\delta)\right) d s<\int_{0}^{t}-2 \kappa d s$, we can 
obtain that $V(\delta(t))<V(\delta(0)) e^{-2 \kappa t}$. Moreover, since $\|\delta\| \leq$ $\left(2 V(0) e^{-2 \kappa t}\right)^{1 / 2}=|\delta(0)| e^{-\kappa t}, \delta(t)$ converges to 0 exponentially fast with speed $\kappa$ as $t \rightarrow+\infty$; this means that consensus can be reached with a convergence speed of $\kappa$.

When we analyse the robustness of the network, every agent is assumed to be affected independently by white noise of the same intensity; thus the dynamic of each node is given by

$$
\dot{x}(t)=-L x(t)+\xi(t)
$$

(see [2]) with $x \in R^{N}$ and where $\xi(t) \in R^{N}$ is a random signal with $E[\xi(t)]=0, E\left[\xi(t)^{T} \xi(\tau)\right]=(\alpha / 2) I_{N} \delta(t-\tau)$, and $E\left[x(0) \xi^{T}(\tau)\right]=0 . \delta(t)$ is the Dirac delta function and $\alpha>0$ is the intensity of the noise.

Let $y(t)=Q x$, where $Q$ satisfies $Q 1_{N}=0, Q Q^{T}=I_{N-1}$, and $Q^{T} Q=I_{N}-(1 / N) 1_{N} 1_{N}^{T}=R$. A measure of the distance from consensus is the dispersion of the system $\|y(t)\|=$ $\left(y^{T}(t) y(t)\right)^{1 / 2}$. Differentiating $y(t)$, we obtain

$$
\dot{y}(t)=-\bar{L} y(t)+Q \xi(t),
$$

where $\bar{L}=Q L Q^{T}$. Since the graph of networks we considered is undirected and connected, all the eigenvalues of $\bar{L}$ are positive real, and hence $-\bar{L}$ is Hurwitz; therefore, (10) convergences exponentially to zero. In [2], the fact that the speed of convergence will be determined by the eigenvalue of $\bar{L}$ with the smallest real part, which is equivalent to the nonzero smallest eigenvalue $\lambda_{2}$ of $L$, has been proved. Therefore, we can also use dynamic (10) to analyse the convergence speed. For better interpreting the relation between dynamic system and the convergence speed of consensus, in this article, we consider the convergence speed under dynamic (5) for each node.

Definition 9 (see [2]). The robustness of the consensus dynamics to white noise inputs is measured by $H=$ $\lim _{t \rightarrow \infty} E(\|y(t)\|)=\lim _{t \rightarrow \infty}\left(\operatorname{tr}\left(E\left[y(t) y(t)^{T}\right]\right)\right)^{1 / 2}$, where $y(t)$ satisfies (10) and $\|y(t)\|$ defines the distance to consensus.

For the state-space system, $\dot{y}=A y+B u$ and $z=C y$, where $A$ is Hurwitz. And the $H_{2}$ norm is $\left[\operatorname{tr}\left(C X C^{T}\right)\right]^{1 / 2}$, where $X$ is the solution of the Lyapunov equation $A X+X A^{T}+$ $B B^{T}=0$. It is familiar to us that the definition of $H$ is equal to the $H_{2}$ norm of system (10) with output equation $z(t)=I y(t)$.

Lemma 10. Suppose $L$ is a Laplacian matrix of a connected undirected graph with eigenvalues $\lambda_{1}=0<\lambda_{2} \leq \lambda_{3} \leq \cdots \leq$ $\lambda_{N}$. Then the $\mathrm{H}_{2}$ norm which characterizes the robustness of system (10) is

$$
H=\left(\sum_{i=2}^{N} \frac{1}{2 \lambda_{i}}\right)^{1 / 2}
$$

Proof. It is well known that any undirected graph will have a symmetric Laplacian matrix, which is trivially normal ([2]), and we have $L^{T} 1^{N}=0$; then $R L=\left(I-(1 / N) 1_{N} 1_{N}^{T}\right) L=$ $L$ is normal. Since $L$ is symmetric, by proposition of [16], the $H_{2}$ norm of network under dynamic (10) is $H=$ $\left(\sum_{i=2}^{N}\left(1 / 2 \operatorname{Re}\left\{\lambda_{i}\right\}\right)\right)^{1 / 2}=\left(\sum_{i=2}^{N}\left(1 / 2 \lambda_{i}\right)\right)^{1 / 2}$.

Remark 11. One well known property related to the $H_{2}$ norm of undirected graph is the effective resistance $K_{f}$, where $K_{f}$ denotes the Kirchhoff index (see $\left.[2,21,22]\right)$ and $K_{f}=$ $N \sum_{j=2}^{N}\left(1 / \lambda_{j}\right)$; it can be related to the power dissipated by the graph. The relation between $K_{f}$ and $H_{2}$ norm is $H=$ $\left(K_{f} / 2 N\right)^{1 / 2}$.

\section{Network Construction and Main Results}

As we mentioned in the introduction part, the star-composed network of this paper is a kind of network in which all nodes have identical dynamics, and it has the topology composed of linking the center nodes among basic star topologies. The links between two nodes mean that they have communication for information. The star-composed structures in this article are all undirected and connected; therefore, by Remark 3, we know that they can achieve consensus. The following procedures (A), (B), and (C) are given to define the three classes of networks.

\section{(A) Construction of Star-Composed Network (1)}

Step 1. Given a star topology with $k$ leaves, each node in the star represents an agent. The node with largest degree in the star has the role of communication center, which is called "center node." The node with degree one in the star is called leaf node.

Step 2. Make $n$ copies of the given star topology. The leaf nodes in each star topology exchange information with the center node of the same star.

Step 3. Link every two of all the center nodes of the $n$ star topologies with each other. It makes the centers in the communication network compose a complete graph.

The topology can be characterized by $K_{n} \circ P_{k}$, and we denote it by network (1). We can see that $n$ and $k$ are both positive integers with $n \geq 2$ and $k \geq 2$; this condition holds for all the networks we constructed in our article. Figure 2 is an example of network (1).

Theorem 12. Let $P_{k}$ be empty graphs with $k$ nodes and $K_{n}$ be a complete graph with $n$ nodes. If the network has the topology of network (1), then the convergence speed of consensus is $\lambda_{2}=$ $\left(n+k+1-\sqrt{(n+k+1)^{2}-4 n}\right) / 2$, and the $H_{2}$ norm of the network is $H=(1 / 2(k+1)+(n-1)(n+k+1) / 2 n+n(k-1) / 2)^{1 / 2}$.

Proof. $\operatorname{Spec}\left(L\left(K_{n}\right)\right)=\left(\begin{array}{cc}0 & n \\ 1 & n-1\end{array}\right)$ (see [19]), and 0 is the eigenvalue of $P_{k}$ with multiplicity $k$.

By Lemma 6, we have

(i) $\left(0+k+1 \pm \sqrt{\left.(k+1)^{2}-4 \times 0\right)} / 2 \in \operatorname{spec}\left(L\left(K_{n}\right.\right.\right.$ 。 $\left.\left.P_{k}\right)\right)$; it follows that 0 and $k+1 \in \operatorname{spec}\left(L\left(K_{n} \circ P_{k}\right)\right)$ with multiplicity 1 ; we can also acquire that $(n+k+$ 


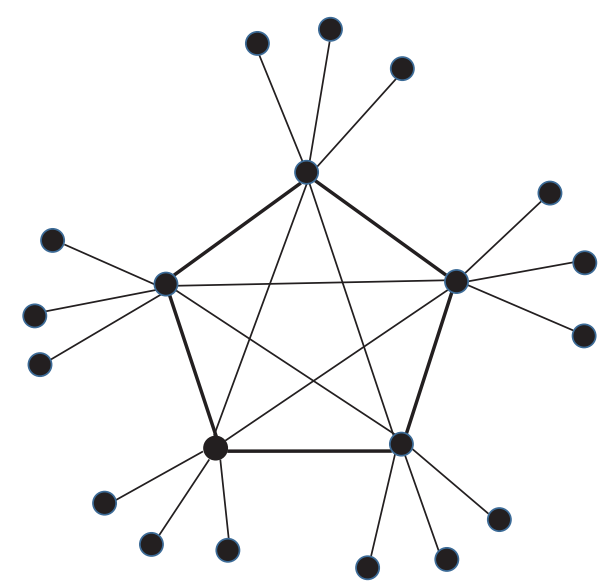

FIgURE 2: The communication topology $K_{5} \circ P_{3}$.

$1 \pm \sqrt{\left.(n+k+1)^{2}-4 n\right)} / 2 \in \operatorname{spec}\left(L\left(K_{n} \circ P_{k}\right)\right)$ with multiplicity $(n-1)$;

(ii) $1 \in \operatorname{spec}\left(L\left(K_{n} \circ P_{k}\right)\right)$ with multiplicity $n(k-1)$; then the total number of eigenvalues of the Laplacian matrix is $2+2(n-1)+n(k-1)=n+n k$, which equals the number of nodes of $K_{n} \circ P_{k}$.

Since $\lambda_{2}$ is the smallest nonzero Laplacian eigenvalue and the eigenvalues of $L$ are nonnegative, $\lambda_{2}=\min \{(n+k+1-$ $\left.\sqrt{\left.(n+k+1)^{2}-4 n\right)} / 2,1\right\}$. Due to the theorem that $\lambda_{2} \leq \delta(G)$ (see [23]), where $\delta(G)$ is the minimum degree of graph $G$, and since the minimum degree of graph of the network is 1 , and it can be proved that $\left(n+k+1-\sqrt{\left.(n+k+1)^{2}-4 n\right)} / 2<1\right.$, therefore, $\lambda_{2}=\left(n+k+1-\sqrt{\left.(n+k+1)^{2}-4 n\right)} / 2\right.$.

Since the graphs of the networks are undirected, their Laplacian matrix is symmetric; by Lemma 10, the $H_{2}$ norm of the network is

$$
\begin{aligned}
H & =\left(\sum_{i=2}^{N} \frac{1}{2 \lambda_{i}}\right)^{1 / 2}=\left(\frac{1}{2(k+1)}\right. \\
+ & +\frac{n-1}{(n+k+1)+\sqrt{(k+1+n)^{2}-4 n}} \\
+ & \\
+ & \left.\frac{n(k-1)}{2}\right)^{1 / 2}=\left(\frac{1}{2(k+1)}\right) \\
+ & \left.\frac{(n-1)(n+k+1)}{2 n}+\frac{n(k-1)}{2}\right)^{1 / 2}
\end{aligned} .
$$

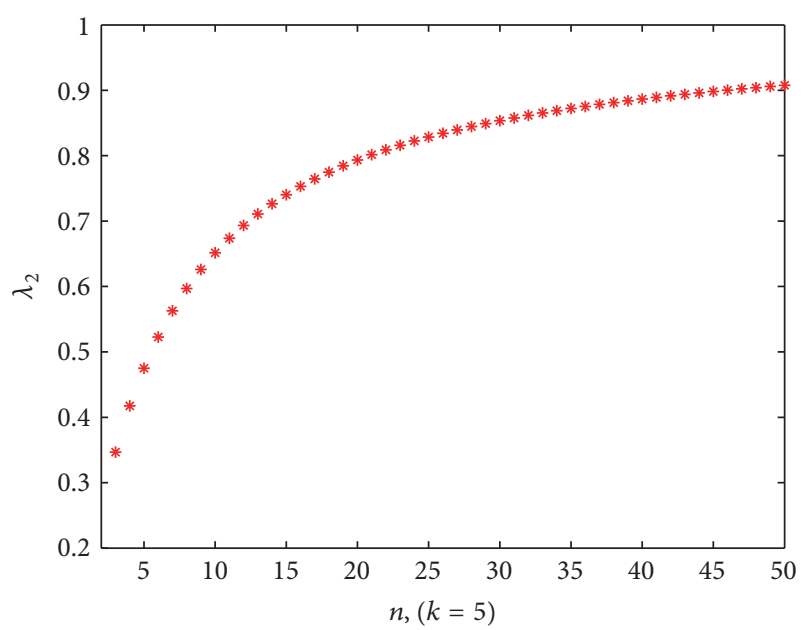

FIgURE 3: The variance of $\lambda_{2}$ for $K_{n} \circ P_{5}$.

Remark 13. Now we discuss the monotonicity of $\lambda_{2}$ and the $\mathrm{H}_{2}$ norm of network (1) when one of $n$ and $k$ is fixed.

\section{The Variance of $\lambda_{2}$}

(i) If $k$ is fixed as a constant, which means the number of leaves of each star is fixed, then let $f(x)=(x+k+$ $\left.1-\sqrt{(x+k+1)^{2}-4 x}\right) / 2$ be a continuously differentiable function; it can be derived that $f^{\prime}(x)>0$; therefore $\lambda_{2}=\left(n+k+1-\sqrt{(n+k+1)^{2}-4 n}\right) / 2$ increases as the number of center nodes $n$ increases.

(ii) If the number of center nodes $n$ is fixed, then let $g(x)=\left(n+x+1-\sqrt{\left.(n+x+1)^{2}-4 n\right)} / 2\right.$ be a continuously differentiable function; it can be derived that $g^{\prime}(x)<0$; therefore, $\lambda_{2}=(n+k+1-$ $\sqrt{\left.(n+k+1)^{2}-4 n\right)} / 2$ decrease as the number of leaf nodes $k$ increases.

\section{The Variance of $\mathrm{H}_{2}$ Norm}

(i) If $k$ is fixed as a constant, let $H(x)=(1 / 2(k+1)+(x-$ $1)(x+k+1) / 2 x+x(k-1) / 2)^{1 / 2}$ be a continuously differentiable function; it can be derived that $H^{\prime}(x)>$ 0 ; therefore, the $\mathrm{H}_{2}$ norm increases as the number of center nodes increases.

(ii) If $n$ is fixed as a constant, let $H(x)=(1 / 2(x+1)+$ $(n-1)(n+x+1) / 2 n+n(x-1) / 2)^{1 / 2}$; it can be derived that $H^{\prime}(x)>0$; therefore, in this case, the $H_{2}$ norm increases as the number of leaf nodes of each star increases.

We give examples for the variance of $\lambda_{2}$ and $H_{2}$ norm of the above cases in Figures 3, 4, 5, and 6.

\section{(B) Construction of Star-Composed Network (2)}

Step 1. Given a star topology with $k$ leaves, each node in the star represents an agent. The node with the largest degree in 


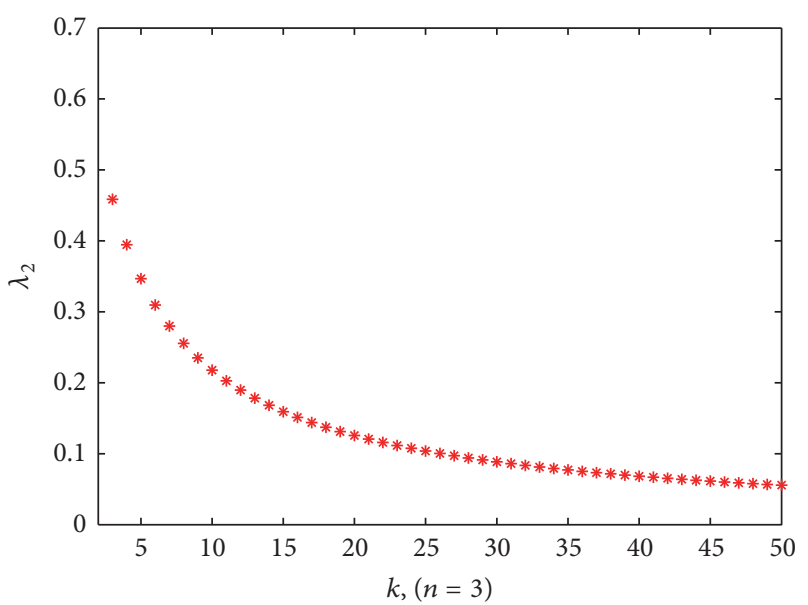

Figure 4: The variance of $\lambda_{2}$ for $K_{3} \circ P_{k}$.

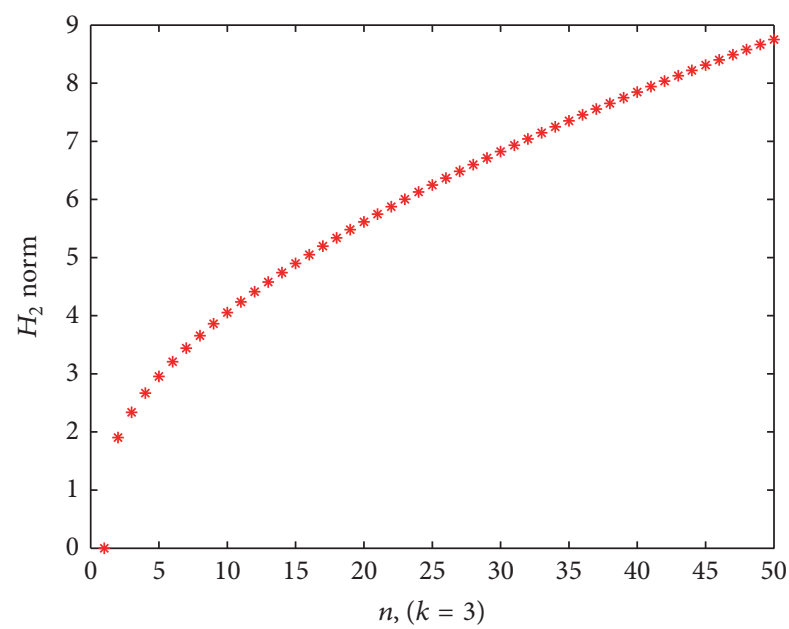

Figure 5: The $H_{2}$ norm for topology $K_{n} \circ P_{3}$.

the star has the role of communication center, which is named center node. The node of degree one in the star is called leaf node.

Step 2. Make $n$ identical copies of the star; separate all the center nodes into two parts such that the center nodes in the same part have no connection with each other.

Step 3. Link each center node in one part to every center node in the other part in order that the center nodes in the same part have the same degree.

The topology which the above procedure made can be characterized by $K_{a, b} \circ P_{k}$ (see Figure 7 ). It can be seen that the topology composed only of the centers of the above procedure is a complete bipartite graph.

For the simplicity and practical meaning of result (see Remark 15), we consider the special case that the number of center nodes in the two parts are equal, and the topology of the special case is denoted by network (2).

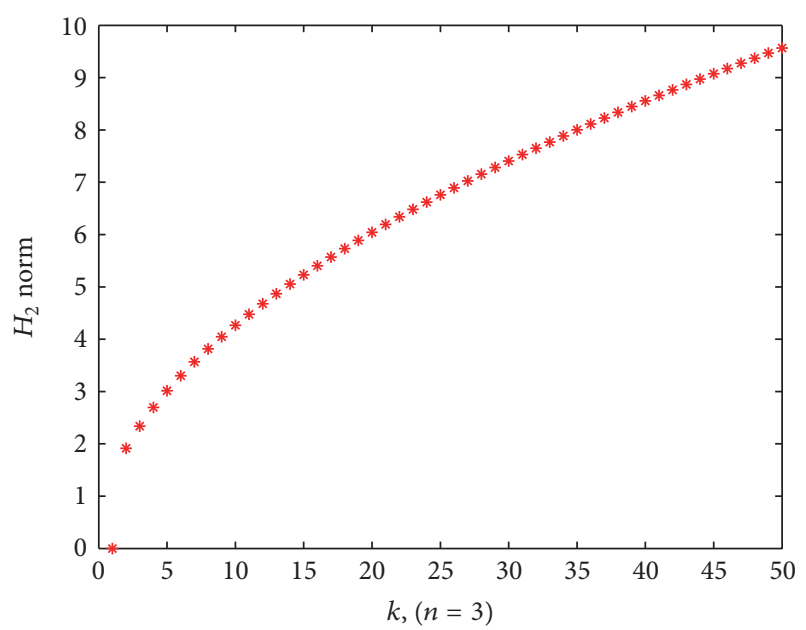

FIgURE 6: The $H_{2}$ norm for topology $K_{3} \circ P_{k}$.

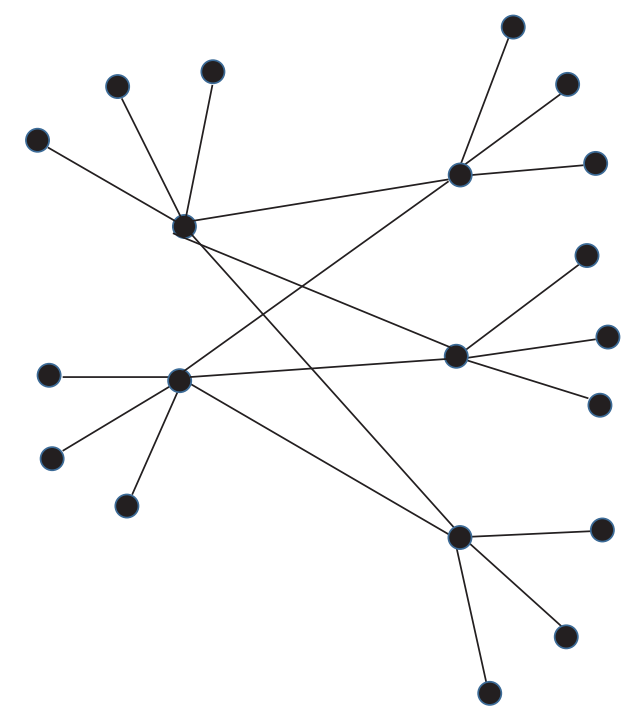

FIgURE 7: The communication topology $K_{2,3} \circ P_{3}$.

Theorem 14. Let $K_{a, b}$ be a complete bipartite graph, where $a, b$ is the number of nodes of each part, and $a, b \geq 2$. Let $P_{k}$ be an empty graph with $k$ nodes. If $a \leq b$, then the convergence speed of the topology $K_{a, b} \circ P_{k}$ is $\lambda_{2}=(a+k+1-$ $\sqrt{\left.(a+k+1)^{2}-4 a\right)} / 2$, and the $H_{2}$ norm which characterizes the robustness is $H=(1 / 2(k+1)+(b-1)(a+k+1) / 2 a+(a-$ $1)(b+k+1) / 2 b+(a+b+k+1) / 2(a+b)+(1 / 2)(a+b)(k-1))^{1 / 2}$.

Proof. Since the Laplacian spectrum of complete bipartite graph is (see [7])

$$
\operatorname{spec}\left(L\left(K_{a, b}\right)\right)=\left(\begin{array}{cccc}
0 & a & b & a+b \\
1 & b-1 & a-1 & 1
\end{array}\right),
$$

by Lemma 6, we have

(i) $\left(k+1 \pm \sqrt{(k+1)^{2}}\right) / 2 \in \operatorname{spec}\left(K_{a, b} \circ P_{k}\right)$; it follows that 0 and $k+1 \in \operatorname{spec}\left(K_{a, b} \circ P_{k}\right)$ with multiplicity 1 , 
$\left(a+k+1 \pm \sqrt{\left.(a+k+1)^{2}-4 a\right)} / 2 \in \operatorname{spec}\left(K_{a, b} \circ P_{k}\right)\right.$ with multiplicity $(b-1),\left(b+k+1 \pm \sqrt{\left.(b+k+1)^{2}-4 b\right)} / 2 \epsilon\right.$ $\operatorname{spec}\left(K_{a, b} \circ P_{k}\right)$ with multiplicity $(a-1)$, and $(a+b+k+$ $\left.1 \pm \sqrt{(a+b+k+1)^{2}-4(a+b)}\right) / 2 \in \operatorname{spec}\left(K_{a, b} \circ P_{k}\right)$ with multiplicity 1 ;

(ii) $1 \in \operatorname{spec}\left(K_{a, b} \circ P_{k}\right)$ with multiplicity $(a+b)(k-1)$.

Set $F(x)=\left((x+k+1)-\sqrt{(x+k+1)^{2}-4 x}\right) / 2$; we can acquire that $F^{\prime}(x)>0$; therefore, to derive the smallest nonzero eigenvalue $\lambda_{2}$ of $L$, we only need to make the comparison between $\left(a+k+1-\sqrt{(a+k+1)^{2}-4 a}\right) / 2,(b+k+1-$ $\left.\sqrt{(b+k+1)^{2}-4 b}\right) / 2$, and 1 . Thus, the convergence speed of consensus of our network is $\lambda_{2}=\min \{1,(a+k+1-$ $\sqrt{\left.(a+k+1)^{2}-4 a\right)} / 2,\left(b+k+1-\sqrt{\left.(b+k+1)^{2}-4 b\right)} / 2\right\}$. Since the algebraic connectivity $\lambda_{2}$ is always less than or equal to the smallest degree, that is, $\lambda_{2} \leq \delta(G)$, it is obvious that in our star-composed topology $\delta(G)=1$; thus we have $\lambda_{2} \leq 1$; therefore, $\lambda_{2}=\left(a+k+1-\sqrt{(a+k+1)^{2}-4 a}\right) / 2$. By Lemma 10, the $\mathrm{H}_{2}$ norm which characterizes the robustness of our network is

$$
\begin{aligned}
H & =\left(\sum_{i=2}^{N} \frac{1}{2 \lambda_{i}}\right)^{1 / 2}=\left(\frac{1}{2(k+1)}\right. \\
+ & +\frac{(b-1)}{a+k+1+\sqrt{(a+k+1)^{2}-4 a}} \\
+ & \frac{(b-1)}{b+k+1+\sqrt{(a+k+1)^{2}-4 a}} \\
+ & \frac{(a-1)}{b+k+1-\sqrt{(b+k+1)^{2}-4 b}} \\
+ & \frac{(a-1)}{a+b+k+1+\sqrt{(a+b+k+1)^{2}-4(a+b)}} \\
+ & \frac{(b-1)(a+k+1)}{2 a+b+1)(b+k+1)} \\
+ & \frac{1}{a+b+k+1-\sqrt{(a+b+k+1)^{2}-4(a+b)}}
\end{aligned}
$$

Remark 15. It is well known that the complete graph has the structure that every node has the same degree, which means each center node has the same communication role of the network. It is familiar to us that each node of undirected cycle has the degree two, and the spectrum of the cycle has already been determined; therefore it has accurate eigenvalues; we can acquire $\lambda_{2}$ from its spectrum.

According to this fact, a natural question arises that if the topology of the regular graph of networks may have the similar analysis of determining $\lambda_{2}$, which can characterize the convergence speed? However, it is a pity that the spectrum of general $k$-regular graph ([18]) has not been determined by anyone yet, where $k \geq 2$ can be any positive integer. We know that the structure of bipartite graph has many applications in the network science ([24]), such as man cooperative network, student course selection network, and online community network. It is interesting to see that the structure which procedure (B) made can be viewed as adhering each node of bipartite graph with a star topology.

Based on the above understanding, to derive a more simple solution of the performance, we consider the case that the linking relations among the centers have the structure of balanced complete bipartite graph, in which each part of the structure has the same number of nodes, which means $a=b$, and we denote this kind of network by network (2). In this special case, all center nodes in the network have the same degree; thus, the following corollary can be derived.

Corollary 16. When the two parts of the network have the same number of center nodes, that is, $a=b$, we have $\operatorname{spec}\left(L\left(K_{a, a}\right)\right)=\left(\begin{array}{ccc}0 & a & 2 a \\ 1 & 2(a-1) & 1\end{array}\right)$, and the convergence speed for the consensus of network (2) with topology $K_{a, a} \circ P_{k}$ equals $\lambda_{2}=$ $\left(a+k+1-\sqrt{(a+k+1)^{2}-4 a}\right) / 2$. Its robustness can be determined by $H=(1 / 2(k+1)+(a-1)(a+k+1) / a+a(k-1)+$ $(2 a+k+1) / 4 a)^{1 / 2}$, where $a \geq 1, k \geq 2$.

Remark 17. With the similar analysis of Theorem 12, it can be deduced that the $\lambda_{2}$ increases as $a$ increases and it decreases as the number of leaves of each star $k$ increases; besides, 1 is an upper bound of $\lambda_{2}$. Next, we discuss the monotonicity of $\mathrm{H}_{2}$ norm:

(i) If $k$ is fixed, then let $H(x)=(1 / 2(k+1)+(x-1)(x+$ $k+1) / x+x(k-1)+(2 x+k+1) / 4 x)^{1 / 2}$. It can be derived that $H^{\prime}(x)>0$; therefore, in this case, the $\mathrm{H}_{2}$ norm increases as $a$ increase.

(ii) If $a$ is fixed, let $H(x)=(1 / 2(x+1)+(a-1)(a+x+$ $1) / a+a(x-1)+(2 a+x+1) / 4 a)^{1 / 2}$. We can derived that $H^{\prime}(x)>0$; therefore, the $H_{2}$ norm increases as $k$ increases.

Numerical examples for the variance of $\lambda_{2}$ and $H_{2}$ norm are given in Figures 8, 9, 10, and 11.

\section{(C) Construction of Star-Composed Network (3)}

Step 1. With the same operation of constructions (A) and (B), given $n$ copies of the given star topology, each star has $k$ leaves. 


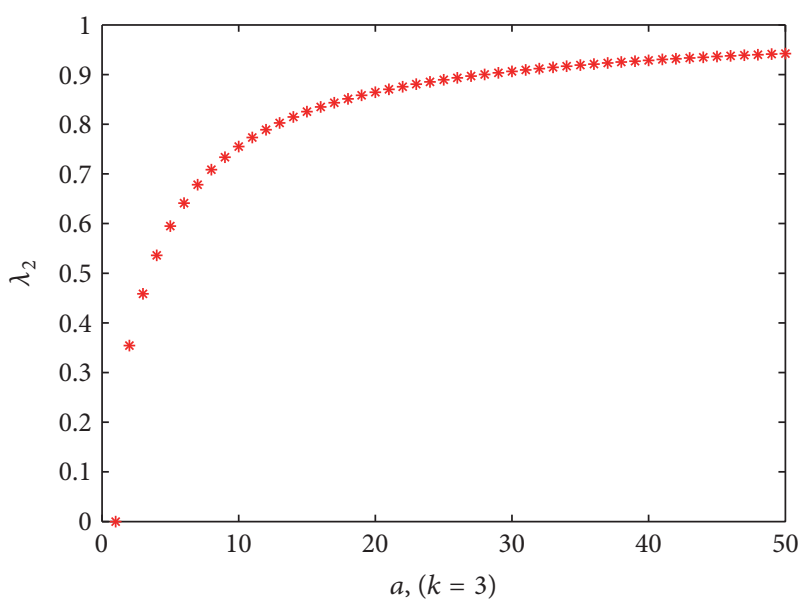

FIgURE 8: The variance of $\lambda_{2}$ for $K_{a, a} \circ P_{3}$.

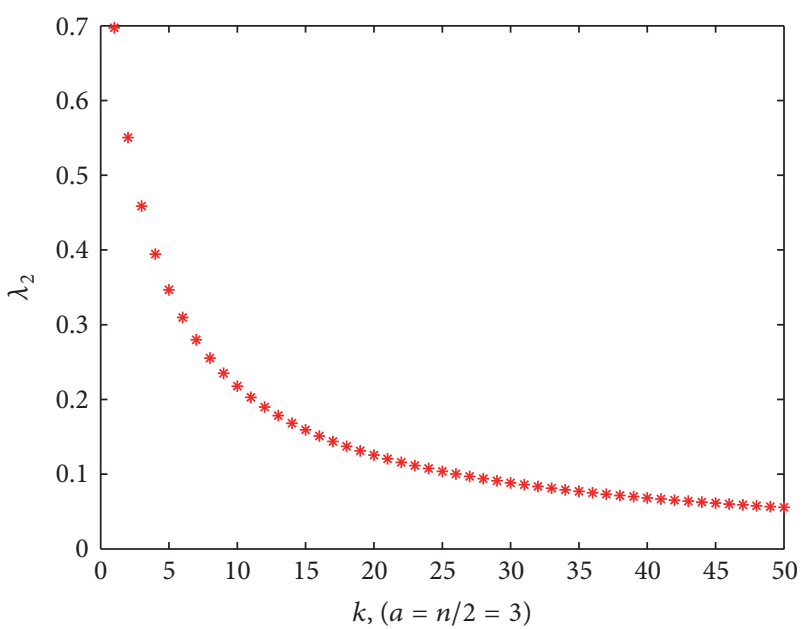

Figure 9: The variance of $\lambda_{2}$ for $K_{3,3} \circ P_{k}$.

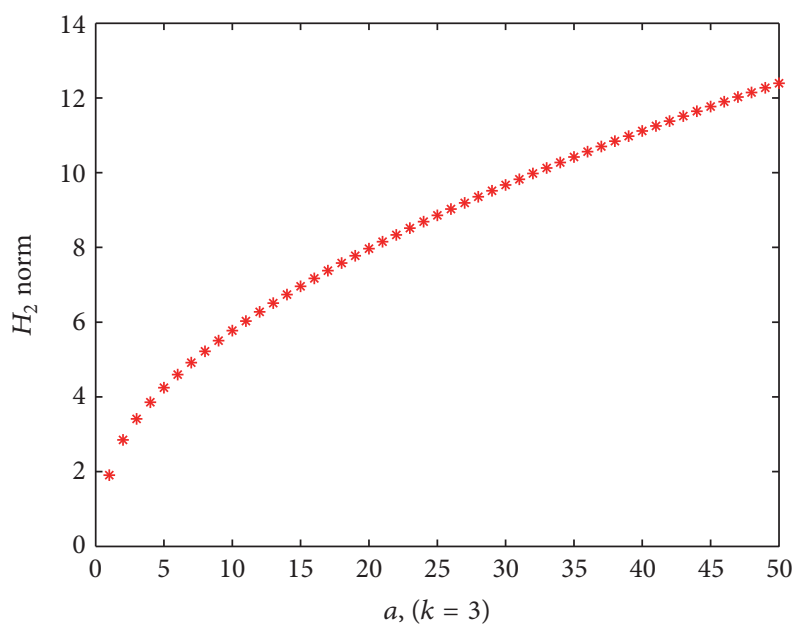

FIgURE 10: The variance of $H_{2}$ norm for $K_{a, a} \circ P_{3}$.

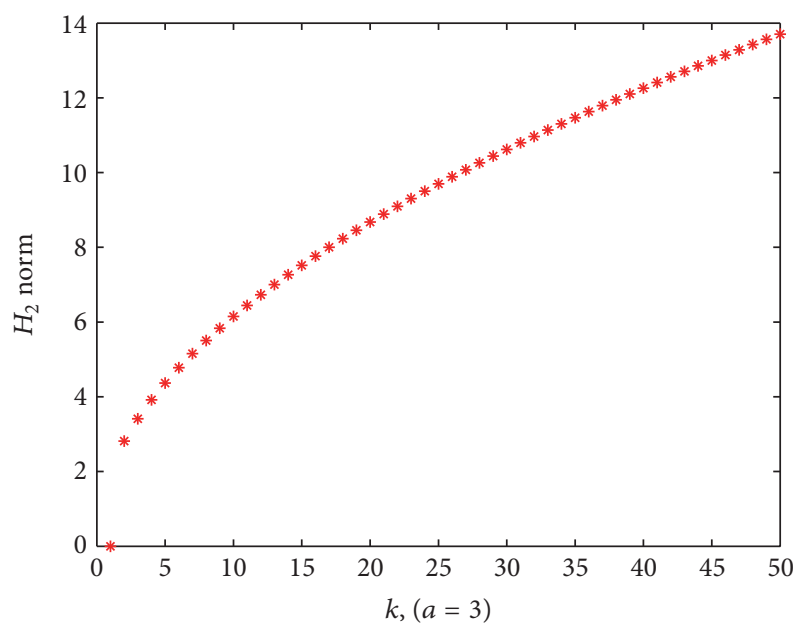

FIgURE 11: The variance of $H_{2}$ norm for $K_{3,3} \circ P_{k}$.

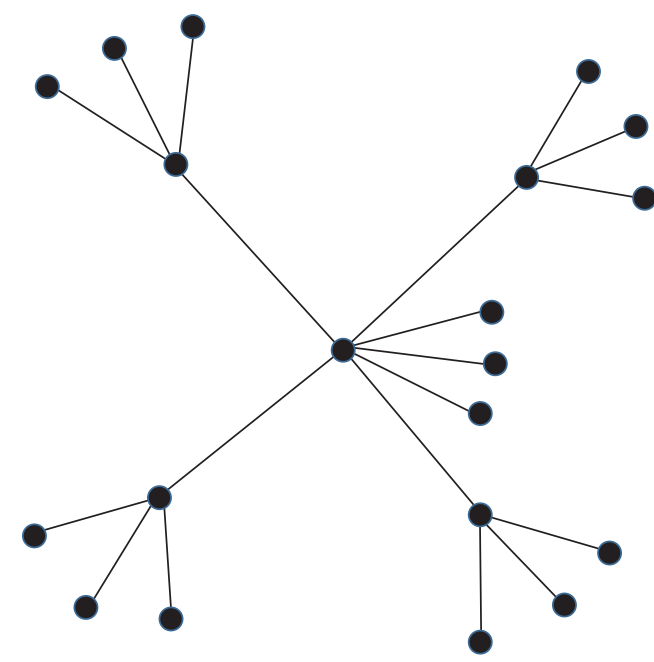

Figure 12: The communication topology $S_{4} \circ P_{3}$.

Step 2. Comprehend each center node of the given stars as an agent of signal center; then choose one of all the centers arbitrarily as the general communication center of the whole network.

Step 3. Link all the other centers to the general center of the network.

The topology can be constructed by the operation of corona and can be expressed by $S_{n-1} \circ P_{k}$, where $S_{n-1}$ is a star with $n-1$ leaves; we denoted the network by network (3). An example of the network is shown in Figure 12.

Theorem 18. The network (3) which is characterized by $S_{n-1}$ 。 $P_{k}$ has the convergence speed $\lambda_{2}=\left(2+k-\sqrt{\left.(k+2)^{2}-4\right)} / 2\right.$, and the $H_{2}$ norm equals $H=(1 / 2(k+1)+(n+k+1) / 2 n+$ $((n-2)(k+2)+n(k-1)) / 2)^{1 / 2}$. 
Proof. It is obvious that $S_{n-1}=K_{1} \circ P_{n-1}$. By Lemma 6, we have

(i) $\left(0+(n-1)+1 \pm \sqrt{n^{2}-4 \times 0}\right) / 2 \in \operatorname{Spec}\left(L\left(K_{1} \circ P_{n-1}\right)\right)$; it follows that $n$ and $0 \in \operatorname{Spec}\left(L\left(K_{1} \circ P_{n-1}\right)\right)$ with multiplicity 1;

(ii) $1 \in \operatorname{spec}\left(L\left(S_{n-1}\right)\right)$ with multiplicity $n-2$; therefore,

$$
\operatorname{spec}\left(L\left(S_{n-1}\right)\right)=\left(\begin{array}{ccc}
0 & 1 & n \\
1 & n-2 & 1
\end{array}\right) .
$$

Next, we calculate the Laplacian spectrum of $S_{n-1} \circ P_{k}$. Again, by Lemma 6, we have

(iii) $\left(k+1 \pm \sqrt{\left.(k+1)^{2}-4 \times 0\right)} / 2 \in \operatorname{spec}\left(L\left(S_{n-1} \circ P_{k}\right)\right)\right.$ with multiplicity 1 ; it follows that 0 and $k+1 \epsilon$ $\operatorname{Spec}\left(L\left(S_{n-1} \circ P_{k}\right)\right)$ with multiplicity $1,(n+k+1 \pm$ $\left.\sqrt{\left.(n+k+1)^{2}-4 \times n\right)}\right) / 2 \in \operatorname{spec}\left(L\left(S_{n-1} \circ P_{k}\right)\right)$ with multiplicity 1 , and $\left(2+k \pm \sqrt{\left.(k+2)^{2}-4\right)}\right) / 2 \epsilon$ $\operatorname{spec}\left(L\left(S_{n-1} \circ P_{k}\right)\right)$ with multiplicity $(n-2)$.

(iv) $1 \in \operatorname{Spec}\left(L\left(S_{n-1} \circ P_{k}\right)\right)$ with multiplicity $n(k-1)$; similar to the analysis of Theorem 12 , set $F(x)=((x+k+1)-$ $\left.\sqrt{(x+k+1)^{2}-4 x}\right) / 2$; since $F(x)^{\prime}>0$, and $k$ is an positive integer, we have that $\left(2+k-\sqrt{\left.(k+2)^{2}-4\right)} / 2\right.$ is the minimum nonzero Laplacian eigenvalue of the graph except 1 . Let $g(y)=\left(2+y-\sqrt{(y+2)^{2}-4}\right) / 2$; it can be derived that $g(y)^{\prime}<0$; therefore, the maximum value of $\lambda_{2}$ equals $g(2)=2-\sqrt{3}<1$; it accords with the result of algebraic graph theory that $\lambda_{2} \leq \delta(G)=1$, where $\delta(G)$ is the minimum degree of graph $G$. Therefore, $\lambda_{2}=\left(2+k-\sqrt{\left.(k+2)^{2}-4\right)} / 2\right.$.

Due to the eigenvalues derived above, the $\mathrm{H}_{2}$ norm of the network equals

$$
\begin{aligned}
H & =\left(\frac{1}{2(k+1)}+\frac{1}{n+k+1+\sqrt{(n+k+1)^{2}-4 n}}\right. \\
& +\frac{1}{n+k+1-\sqrt{(n+k+1)^{2}-4 n}} \\
& +\frac{(n-2)}{(k+2)+\sqrt{(k+2)^{2}-4}} \\
& \left.+\frac{(n-2)}{(k+2)-\sqrt{(k+2)^{2}-4}}+\frac{n(k-1)}{2}\right)^{1 / 2} \\
& =\left(\frac{1}{2(k+1)}+\frac{n+k+1}{2 n}\right. \\
+ & \left.\frac{(n-2)(k+2)+n(k-1)}{2}\right)^{1 / 2} .
\end{aligned}
$$

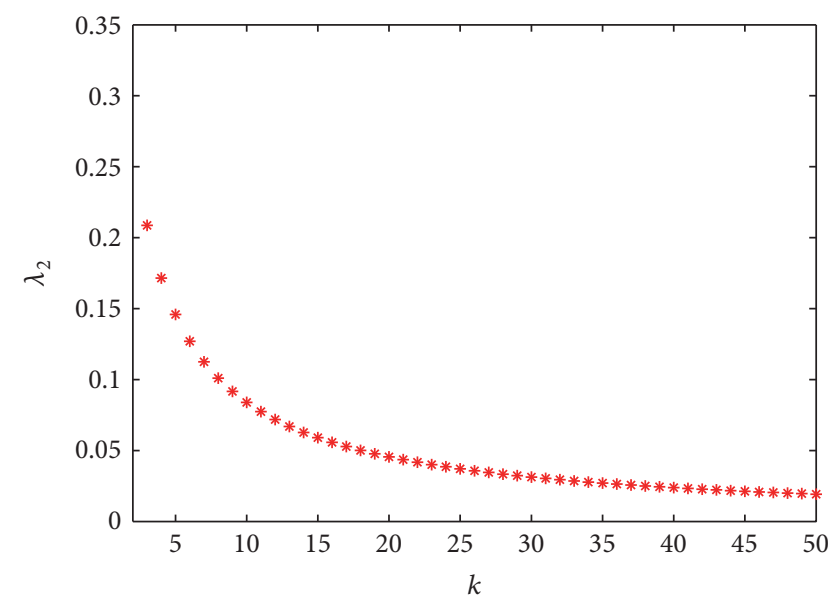

FIGURE 13: The convergence speed for $S_{n-1} \circ P_{k}$.

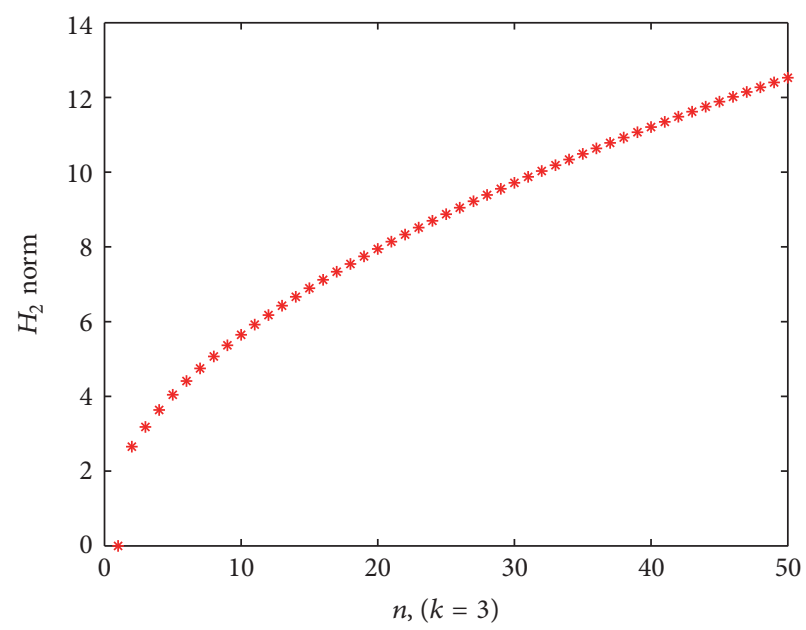

FIGURE 14: The variance of $H_{2}$ norm for $S_{n-1} \circ P_{3}(n \geq 3)$.

Remark 19. Through the derivation of Theorem 18, we can obtain that $\lambda_{2}$ is monotonically decreasing as $k$ increases.

The variance of $\mathrm{H}_{2}$ norm is discussed as follows:

(i) If $k$ is fixed, let $H(x)=(1 / 2(k+1)+(x+k+1) / 2 x+$ $((x-2)(k+2)+x(k-1)) / 2)^{1 / 2}$; then we can derive that $H(x)^{\prime}>0$; therefore, the $H_{2}$ norm increases as the number of center nodes increases.

(ii) If $n$ is fixed, let $H(x)=(1 / 2(x+1)+(n+x+1) / 2 n+$ $((n-2)(x+2)+n(x-1)) / 2)^{1 / 2}$; then we have $H(x)^{\prime}>0$; therefore, the $\mathrm{H}_{2}$ norm increases as $k$ increase.

Examples of variance for convergence speed and robustness are given in Figures 13, 14, and 15.

Remark 20. In Theorem 18, if there exist $k+1$ identical star topologies, which equal the number of center nodes of the network, that is, $n=k+1$ (see Figure 16), from Example 5 and Figure 16, we find that the network construction method 


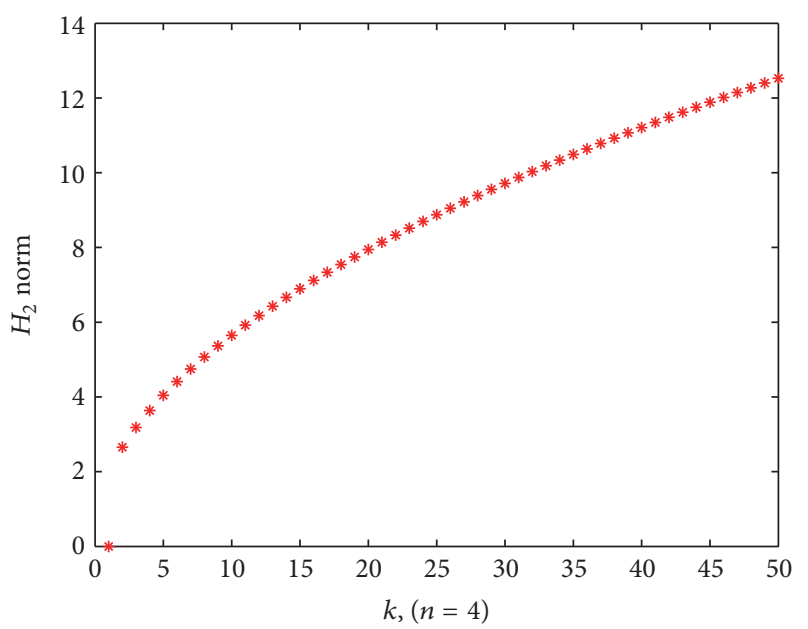

FIGURE 15: The variance of $H_{2}$ norm for $S_{3} \circ P_{k}(k \geq 2)$.

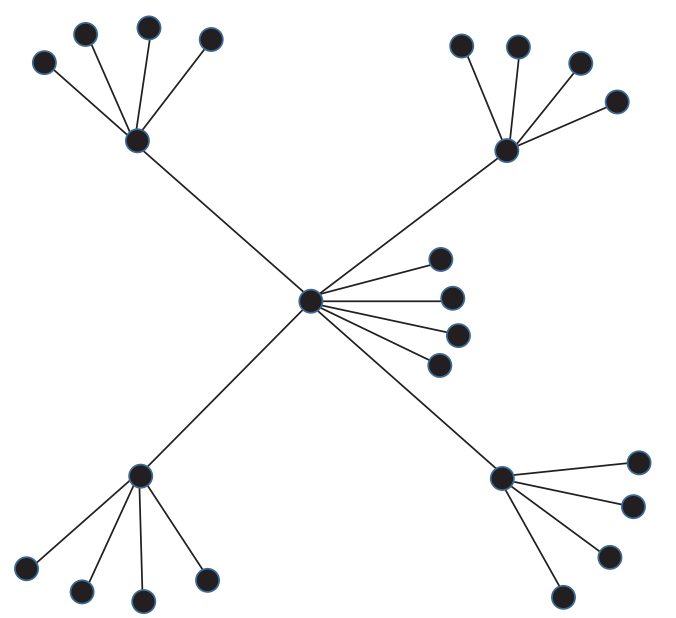

FIgURE 16: The communication topology $S_{4} \circ S_{4}(n=5, k=4)$.

of corona of two graphs can generate a class of small-world polymer networks (see [5]), and Figure 16 is an example of the 2 iterations of the network construction method in [5]. The following corollary can be derived.

Corollary 21. Under the network topology of Remark 20, the convergence speed equals $\lambda_{2}=\left(1+n-\sqrt{(n+1)^{2}-4}\right) / 2$, and the $H_{2}$ norm is $H=(1 / 2 n+1+(n-2)(1+2 n) / 2)^{1 / 2}$.

Proof. By Lemma 6, with the similar analysis of the above theorem, we have

(i) $\left(n \pm \sqrt{n^{2}}\right) / 2 \in \operatorname{spec}\left(L\left(S_{k} \circ P_{k}\right)\right)$; it follows that 0 and $n \in \operatorname{spec}\left(L\left(S_{k} \circ P_{k}\right)\right)$ with multiplicity $1 ;(2 n \pm$ $\sqrt{\left.(2 n)^{2}-4 n\right)} / 2 \in \operatorname{spec}\left(L\left(S_{k} \circ P_{k}\right)\right)$ with multiplicity $1 ;\left(1+n \pm \sqrt{\left.(n+1)^{2}-4\right)} / 2 \in \operatorname{spec}\left(L\left(S_{k} \circ P_{k}\right)\right)\right.$ with multiplicity $(n-2)$;

(ii) $1 \in \operatorname{spec}\left(L\left(S_{k} \circ P_{k}\right)\right)$ with multiplicity $n(n-2)$; since there exist at least two star clusters, which means $n \geq$

$$
\begin{aligned}
& 2 \text {, and because } 1+n+\sqrt{(n+1)^{2}-4}>2 \text {, let } f(x)= \\
& 2 x-\sqrt{4 x^{2}-4 x} \text {; we can derive that } f(x)^{\prime}>0 \text {; thus } \\
& 2 n-\sqrt{4 n^{2}-4 n} \geq 2 \text {; therefore } \\
& \frac{\left(\left(2 n-\sqrt{4 n^{2}-4 n}\right) / 2\right)}{\left(\left(1+n-\sqrt{(n+1)^{2}-4}\right) / 2\right)} \\
& =\frac{\left(2 n-\sqrt{4 n^{2}-4 n}\right)\left(1+n+\sqrt{(n+1)^{2}-4}\right)}{4}>1 . \\
& H=\left(\frac{1}{2 n}+\frac{1}{2 n+\sqrt{4 n^{2}-4 n}}+\frac{1}{2 n-\sqrt{4 n^{2}-4 n}}\right. \\
& +\frac{(n-2)}{1+n+\sqrt{(n+1)^{2}-4}}+\frac{(n-2)}{1+n-\sqrt{(n+1)^{2}-4}} \\
& \left.+\frac{n(n-2)}{2}\right)^{1 / 2}=\left(\frac{1}{2 n}+1+\frac{(n-2)(1+2 n)}{2}\right)^{1 / 2} .
\end{aligned}
$$

Remark 22. Let $F(x)=(1 / 2 x+1+(x-2)(1+2 x) / 2)^{1 / 2}$; we can derive that $F^{\prime}(x)>0$; therefore, the $H_{2}$ norm increases as the number of center nodes increases. Due to the proof of Corollary 21, it can be seen that $\lambda_{2}$ decreases as $n$ increases. The variance of $\lambda_{2}$ and $H_{2}$ norm (when $n=2, \ldots, 50 ; k=$ $2, \ldots, 49)$ is showed in Figures 17 and 18.

\section{Simulation Analysis and Comparison}

Now we make the comparison of performance for the starcomposed networks (1)-(3). By the analysis of the above theorems, it can be derived that the monotonicity of function $f(x)=\left(x+k+1-\sqrt{(x+k+1)^{2}-4 x}\right) / 2$ is monotonically increasing. Therefore, when $n$ and $k$ of the three classes of networks are equal, the convergence speed of networks (1)-(3) has the relation $\lambda_{2}^{(1)} \geq \lambda_{2}^{(2)}>\lambda_{2}^{(3)}$, where $\lambda_{2}^{(i)}$ denotes the smallest nonzero Laplacian eigenvalue of the graph of network $(i), i=1,2,3$. We can see that the network (1) has the largest convergence speed of the three, and $\lambda_{2}^{(3)}$ is irrelevant with the number of center nodes $n$. Actually, we have the theorem in the algebraic graph theory that $\lambda_{2}(G) \leq \lambda_{2}\left(G^{\prime}\right)$, where $G^{\prime}$ is the graph obtained by adding an edge to graph 


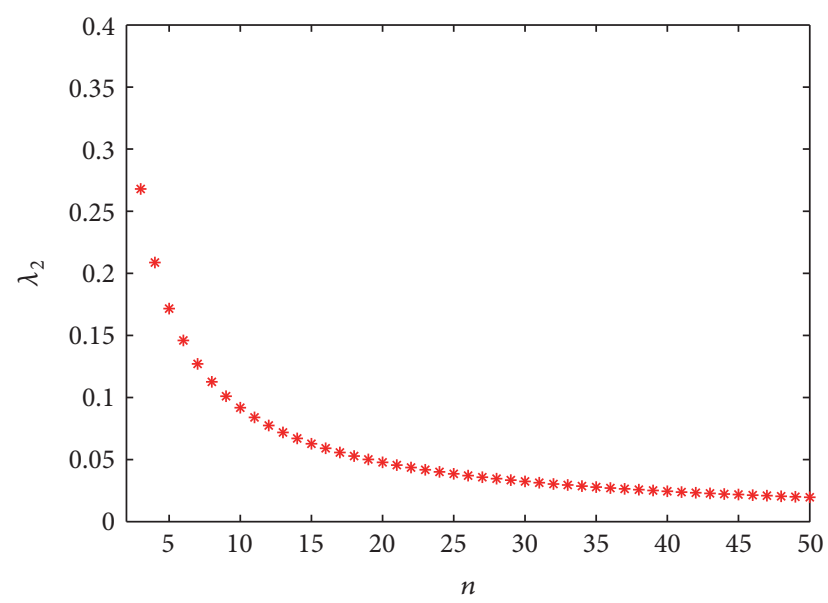

FIgURE 17: $\lambda_{2}$ of $S_{n-1} \circ P_{n-1}(n \geq 3, k=n-1)$.

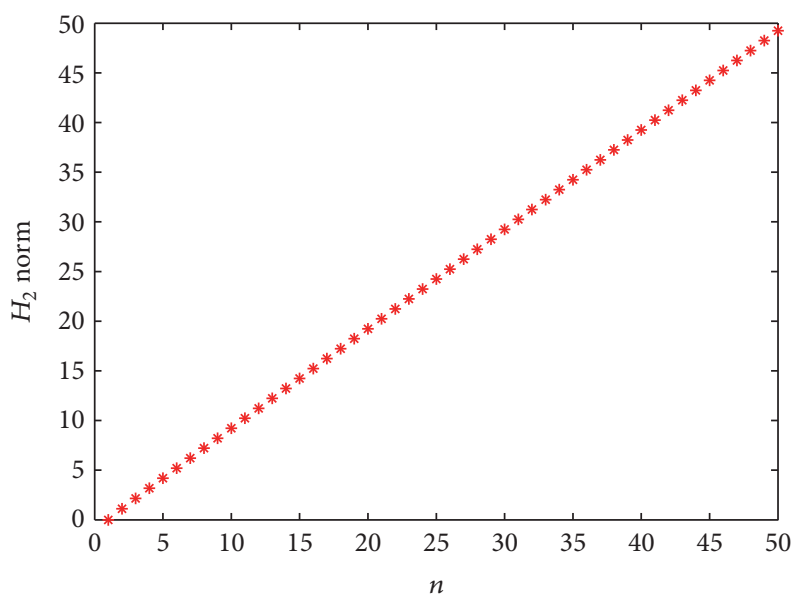

FIgURE 18: The $H_{2}$ norm of $S_{n-1} \circ P_{n-1}(n \geq 3)$.

$G$ (see [23]). Hence, if the linking structure of center nodes of our network has less edges than complete graph $K_{n}$, then the convergence speed of the whole network is always smaller than network (1). The method of constructing the network topology in this article can obviously create a class of smallworld networks by the corona of a sequence of graphs; for instance, the corona $K_{1} \circ P_{n} \circ P_{n} \circ \cdots \circ P_{n}$ is a kind of smallworld polymer network (see Figure 1 in [5]). We can view the corona of these graphs as a construction method of network grown.

Due to the simulation data, it can be seen that the variance of the $H_{2}$ norms of all our networks increases as the number of center nodes $n$ or the number of leaf nodes $k$ is fixed, and the monotonicity of $\mathrm{H}_{2}$ norm of Corollary 21 is similar to the linear relation. Through the construction of the networks, note that $n$ and $k$ satisfy the equation $n(1+k)=N$, where $N$ is the number of nodes of the whole network. When $N$ is fixed, for better comparing these different classes of networks, we describe their variance of $\mathrm{H}_{2}$ norm in the three-dimensional space in Figures 19 and 20.

Through the two numerical examples in Figures 19 and 20 , we find that when $N=100$, the $H_{2}$ norm of network (1)

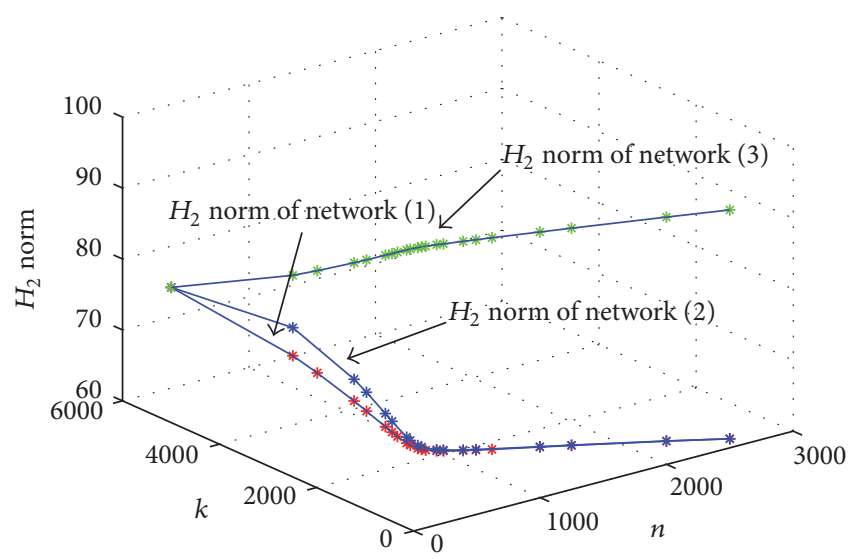

FIGURE 19: The variance of $H_{2}$ norm for network (i) when $N=$ $10000, i=1,2,3$.

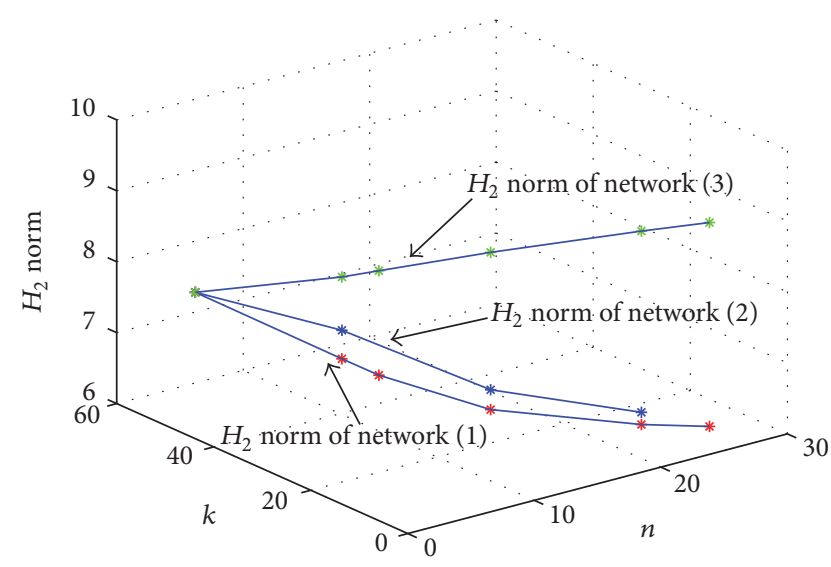

FIGURE 20: The variance of $H_{2}$ norm for network $(i)$ when $N=100$, $i=1,2,3$.

is the smallest of the three. The $\mathrm{H}_{2}$ norm of network (1) and network (2) decreases as $n$ increases. And with $N$ increases, the $\mathrm{H}_{2}$ norm of network (1) and network (2) tend to be the same value. It tells us that when the number of the nodes of the whole network $N$ is fixed, the robustness of network (1) and network (2) tend to be the same as $n$ increase, and since the difference between the two networks is that network (2) has less edges than network (1), therefore, by (3), the cost of network (2) is less than network (1).

When $\mathrm{N}$ is fixed, the $\mathrm{H}_{2}$ norm of network (3) increases as $n$ increases. We can see that when $n=2$, the $H_{2}$ norms of the three classes of star-composed networks are the same; it accords with the fact that they have the same topology when $n=2$ (Figure 21).

When the $n$ identical star topologies are given, by (4), we can obtain that $\rho_{1}=(1 / 2) n(n-1) /(1 / 2) n(n-1)=1, \rho_{2}=$ $(n / 2 \cdot n / 2) /(1 / 2) n(n-1)$ (here we suppose $n / 2$ is an integer), and $\rho_{3}=(n-1) /(1 / 2) n(n-1)$, where $\rho_{i}$ denotes the density of the graph composed only of the center nodes of network (i), $i=1,2,3$. It can be derived that $\rho_{1}>\rho_{2}>\rho_{3}$. Due to the network construction and the simulation figures, we give a conjecture that the $\mathrm{H}_{2}$ norm of network $(i)$ is relevant with 


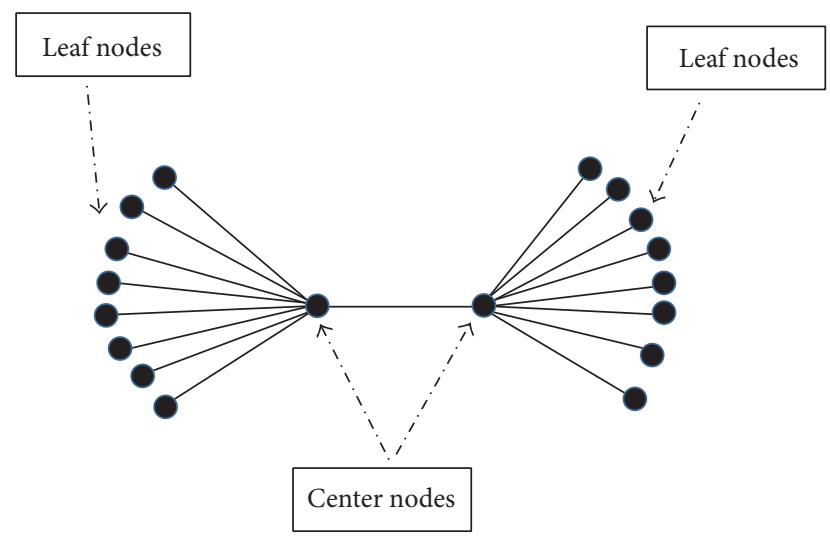

FIGURE 21: Star-composed network $(n=2, k=8)$.

the density $\rho_{i}$; that is, the $H_{2}$ norm decreases as $\rho_{i}$ increases when the same $n$ copy of star clusters is fixed, which means the robustness becomes better as the density of the graph constructed by centers increases.

\section{Conclusion}

Consider synthetically the convergence speed, robustness, and the cost of the network, we find that the topology of network (3) has the largest $\mathrm{H}_{2}$ norm of the three; therefore its robustness is the worst of them; however, it has the smallest cost of the three. Network (2) has an advantage that it has relatively large convergence speed and its robustness is as good as network (1) when the size of the network increases, but its cost is relatively small. Therefore, network (2) has reference values for real applications.

\section{Competing Interests}

The authors declare that they have no competing interests.

\section{Acknowledgments}

The authors express their sincere gratitude to the people who gave them valuable comments. This work was supported by National Natural Science Foundation of Peoples Republic of China (NSFC) (Grants no. 61164004, no. 61473244, and no. 11402223), the National Natural Science Foundation of Xinjiang (NSFXJ) (no. 2015211B005), and NSFC (no. 11301452).

\section{References}

[1] R. Olfati-Saber and R. M. Murray, "Consensus problems in networks of agents with switching topology and time-delays," Institute of Electrical and Electronics Engineers. Transactions on Automatic Control, vol. 49, no. 9, pp. 1520-1533, 2004.

[2] G. F. Young, L. Scardovi, and N. E. Leonard, "Robustness of noisy consensus dynamics with directed communication," in Proceedings of the American Control Conference (ACC '10), pp. 6312-6317, July 2010.
[3] W. Yu, J. Lü, X. Yu, and G. Chen, "Distributed adaptive control for synchronization in directed complex networks," SIAM Journal on Control and Optimization, vol. 53, no. 5, pp. 29803005, 2015.

[4] L. Ji, Q. Liu, and X. Liao, "On reaching group consensus for linearly coupled multi-agent networks," Information Sciences, vol. 287, pp. 1-12, 2014.

[5] H. Liu and Z. Zhang, "Laplacian spectra of recursive treelike small-world polymer networks: analytical solutions and applications," Journal of Chemical Physics, vol. 138, no. 11, 2013.

[6] S. Patterson and B. Bamieh, "Consensus and coherence in fractal networks," IEEE Transactions on Control of Network Systems, vol. 1, no. 4, pp. 338-348, 2014.

[7] W. Sun, Q. Ding, J. Zhang, and F. Chen, "Coherence in a family of tree networks with an application of Laplacian spectrum," Chaos, vol. 24, no. 4, Article ID 043112, 2014.

[8] W. Xiao and I. Gutman, "Resistance distance and Laplacian spectrum," Theoretical Chemistry Accounts, vol. 110, no. 4, pp. 284-289, 2003.

[9] M. Chen and D. Zhou, "Synchronization in uncertain complex networks," Chaos, vol. 16, no. 1, Article ID 013101, pp. 298-311, 2006.

[10] A. E. Kamal, "Star local area networks: A Performance Study," IEEE Transactions on Computers, vol. C-36, no. 4, pp. 483-499, 1987.

[11] R. Martínez-Guerra, C. D. Cruz-Ancona, and C. A. PérezPinacho, "Generalized multi-synchronization viewed as a multi-agent leader-following consensus problem," Applied Mathematics and Computation, vol. 282, pp. 226-236, 2016.

[12] A. A. Rescigno, "Optimally balanced spanning tree of the star network," IEEE Transactions On Computers, vol. 50, no. 1, pp. 88-91, 2001.

[13] M. Porfiri and F. Fiorilli, "Node-to-node pinning control of complex networks," Chaos, vol. 19, no. 1, Article ID 013122, 2009.

[14] J. Wang and Y. Zhang, "Network synchronization in a population of star-coupled fractional nonlinear oscillators," Physics Letters A, vol. 374, no. 13-14, pp. 1464-1468, 2010.

[15] S. J. S. Theesar, M. R. K. Ariffin, and S. Banerjee, "Synchronization and a secure communication scheme using optical star network," Optics and Laser Technology, vol. 54, pp. 15-21, 2013.

[16] S. Barik, S. Pati, and B. K. Sarma, "The spectrum of the corona of two graphs," SIAM Journal on Discrete Mathematics, vol. 21, no. 1, pp. 47-56, 2007.

[17] Q. Liu, "The Laplacian spectrum of Corona of two graphs," Kragujevac Journal of Mathematics, vol. 38, no. 1, pp. 163-170, 2014.

[18] J. Bondy and U. Murty, Graph Theory, Springer, 2008.

[19] D. Cvetković, P. Rowlinson, and S. Simić, An introduction to the theory of graph spectra, vol. 75 of London Mathematical Society Student Texts, Cambridge University Press, Cambridge, 2010.

[20] R. A. Horn and C. R. Johnson, Matrix Analysis, Cambridge University Press, 2nd edition, 2013.

[21] W. Abbas and M. Egerstedt, "Robust graph topologies for networked systems," in Proceedings of the 3rd IFAC Workshop on Distributed Estimation and Control in Networked Systems (NECSYS '12), pp. 85-90, Santa Barbara, Calif, USA, September 2012.

[22] X. Qi, B. Zhou, and Z. Du, "The Kirchhoff indices and the matching numbers of unicyclic graphs," Applied Mathematics and Computation, vol. 289, pp. 464-480, 2016. 
[23] C. Godsil and G. Royle, Algebraic Graph Theory, vol. 207 of Graduate Texts in Mathematics, Springer, New York, NY, USA, 2001.

[24] Z. Li, R.-S. Wang, S. Zhang, and X.-S. Zhang, "Quantitative function and algorithm for community detection in bipartite networks," Information Sciences, vol. 367-368, pp. 874-889, 2016. 


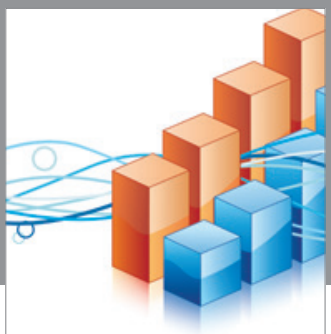

Advances in

Operations Research

vatem alat4

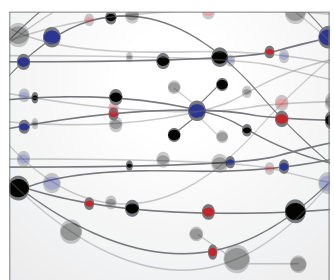

\section{The Scientific} World Journal
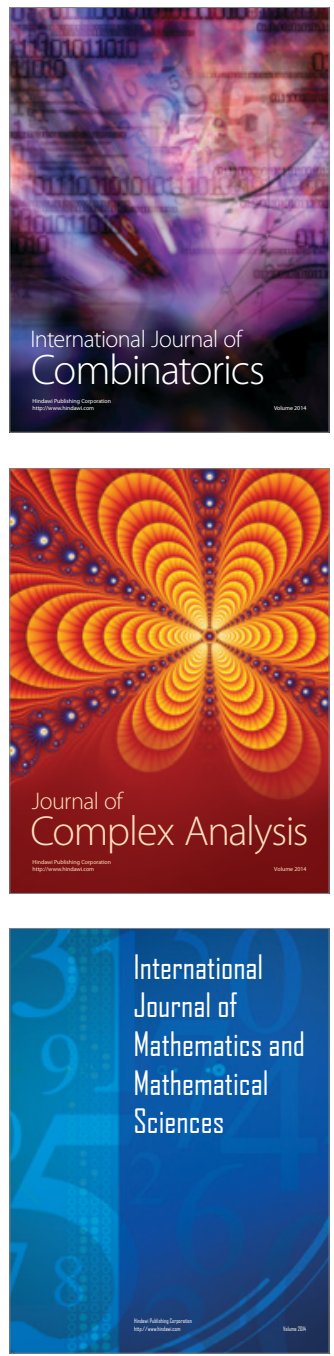
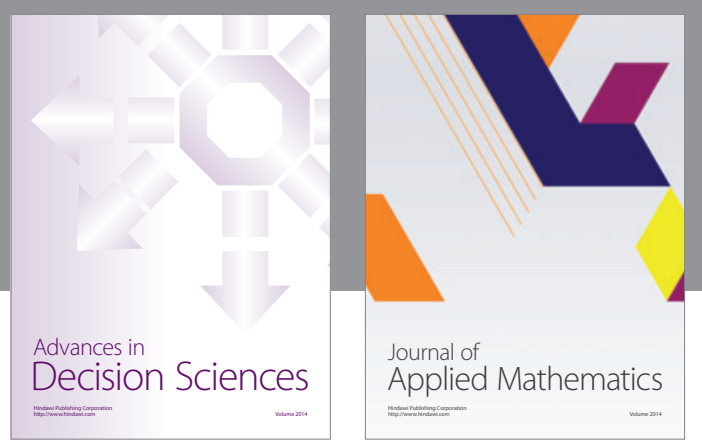

Algebra

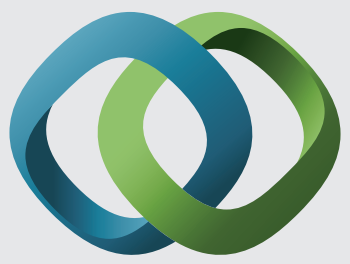

\section{Hindawi}

Submit your manuscripts at

https://www.hindawi.com
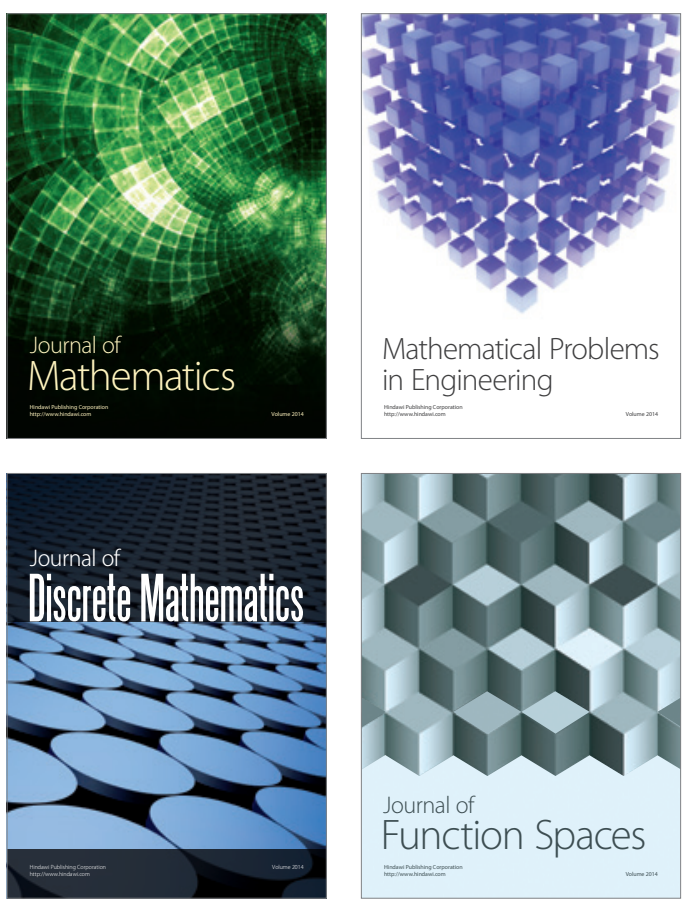

Mathematical Problems in Engineering
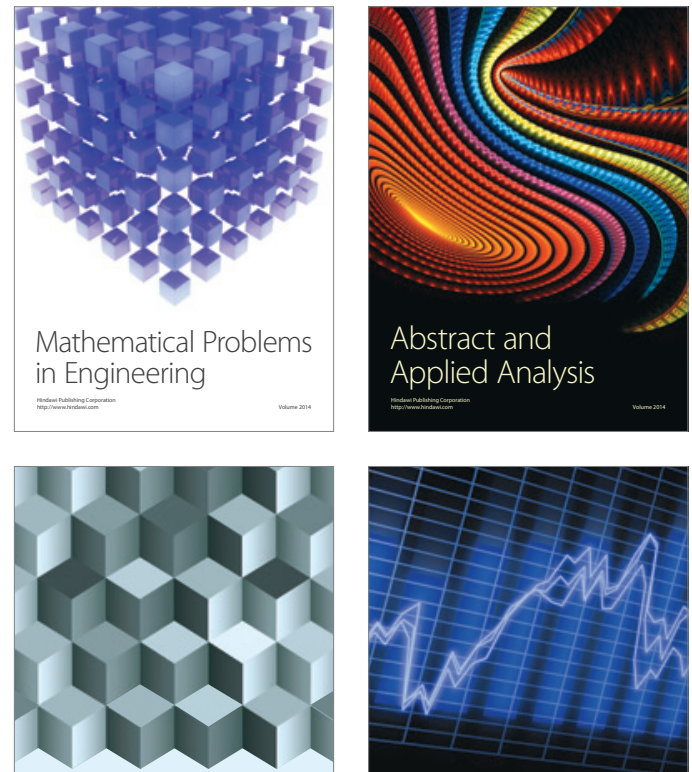

Journal of

Function Spaces

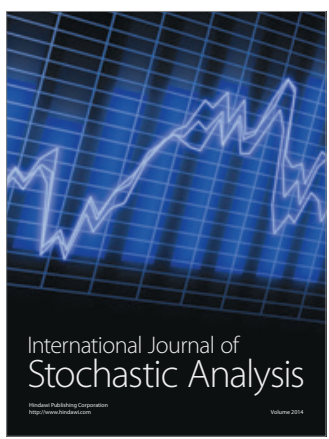

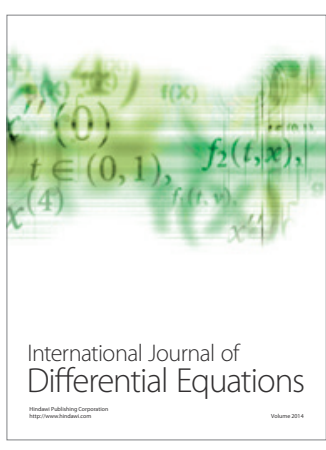
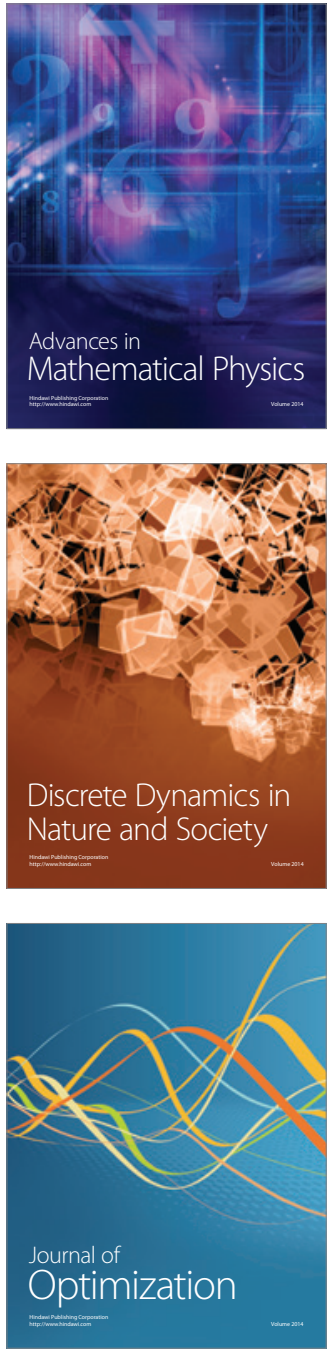